\title{
Comparison of fitting stability of the different soft toric contact lenses
}

\begin{abstract}
Purpose: To compare lens orientation and rotational recovery of five currently available soft toric lenses.

Methods: Twenty subjects were recruited and trialed with each of the study lenses in a random order. Study lenses were PureVision® Toric (B\&L), Air Optix® for Astigmatism (Alcon), Biofinity ${ }^{\circledR}$ Toric (CooperVision), Acuvue ${ }^{\circledR}$ Advance for Astigmatism (Vistakon), and Proclear ${ }^{\circledR}$ Toric (CooperVision). Lens orientation in primary position to determine the lens rotation form the vertical position and rotational recovery to primary gaze orientation following a $45^{\circ}$ manual misorientation for the different lenses was compared.

Results: The Biofinity Toric showed the lowest rotation from the vertical position and the Proclear Toric the highest. Also, the highest and the lowest reorientation speed were related to the Biofinity Toric and the Acuvue Advance for Astigmatism, respectively. The Repeated Measures ANOVA showed a significant difference in the lens rotation $(P=0.004)$ and rotational recovery $(P<0.001)$ among different contact lenses and the performed multiple comparisons indicated differences in rotation and also in reorientation speed were only seen between the Biofinity Toric when compared to four other lenses $(P<0.05)$.
\end{abstract}

\section{Conclusion:}

Although there was appropriate fitting, based upon lens orientation and reorientation speed, with each of the study lenses it would appear that the optimized ballast technique used in the design of the Biofinity Toric helps reduce lens rotation and improve rotational recovery compared to others.

Key words: Contact Lens, Toric Lens, Lens design, Lens orientation, Rotational recovery, Reorientation speed.

\section{Introduction}


Approximately $50 \%$ of patients have significant astigmatism ( $\geq 0.75 \mathrm{DC})$ [1] and about one-third of contact lens patients need astigmatic correction. These patients are sometimes fitted with spherical lenses, resulting in less than optimal vision correction, so toric soft contact lenses can use as a corrective option in this group of patients.[2] Factors influencing toric soft contact lenses fit can be split into patient related factors and the contact lens related ones. The patient related factors can include palpebral aperture, lids position, lid tightness, inter-canthal angle, refractive errors, horizontal visible iris diameter and corneal topography. The factors related to the contact lens include fitting profile (optimal, steep, flat), lens movement after blink, lens modulus and toric soft lens stabilization design.[3, 4]

Although many advances have been made in toric contact lens development, lens rotation continues to affect vision and can occur when the patient blinks or rubs their eyes. Stabilization techniques used in the design of the toric soft lens rely on the interaction between lids and the lens to achieve stabilization and resultantly the lens axis coincide as much as possible to the axis of astigmatism of the eye and the lens rotation minimize post-blinking.[5, 6] These designs include two commonly used methods; Prism-ballasted design that involve interaction primarily from the upper lid and Non prism-ballasted design which both eyelids play an active role in stabilization. $[6,7]$

The stabilization methods used in some of the toric soft contact lenses are: Prism ballast (Pure Vision Toric), Precision balance (Air optix for astigmatism), Prism ballast (Proclear Toric), Optimized Ballast Design or Equi ballast design or Horizontal iso-thickness design (Biofinity Toric), Accelerated stabilization design (ASD) (Acuvue Advance for Astigmatism).[8] The first Four lenses have prism stabilized designs, two of which (Air Optix for Astigmatism (Alcon) and Biofinity Toric (CooperVision)) incorporate modifications to avoid interaction with the bottom lid. The other lens has a dual-thin design with centrally placed stabilization zones.[8]

\section{Methods}


In this study, participants were 20 students of Zahedan University of Medical Sciences (mean age of $21.4 \pm 2.0$ years) who were experienced soft contact lens wearers.

The nature, purposes and methods of this research work were clearly explained to the participants in advance, and their voluntary cooperation and informed consent was obtained from all participants. The study was conducted in accordance with the tenets of the Declaration of Helsinki. The study was approved by the University ethics committee.

Those who met inclusion criteria were entered into the study. The inclusion criteria included:

- Experienced soft contact lens wearers

- Normal tear quality and quantity. Tears were assessed with Tear Break-Up Time (TBUT) (normal results: 15 seconds or greater [19]) and tear prism height (normal results: 0.2-0.4 $\mathrm{mm}$ at the center [9])

- No corneal infiltration and infection

- No pathology in lids and conjunctiva (bulbar and tarsal)[10]

- Normal external anatomical position of the lids

- Refractive errors in a range of plano to -6.00 dioptres (mean spherical equivalent)[6].

The exclusion criteria were:

- Presence of irregular astigmatism

- Cases suspected to have keratoconus using the Pentacam (Oculus Optikgeräte $\mathrm{GmbH}$, Germany)

- History of refractive surgery

- Presence of systemic diseases such as diabetes, connective tissue involvementsSubjects taking medications which could interfere with contact lens wear such as antihistamines, antidepressants and oral contraceptives.[10] 
Refractive errors and the horizontal visible iris diameter only in the right eye were determined using a Topcon KR.8800 autokeratorefractometer (Topcon, Japan). A millimeter ruler was used to measure the vertical palpebral aperture.

Study lenses were PureVision ${ }^{\circledR}$ Toric (Bausch and Lomb), Air Optix ${ }^{\circledR}$ for Astigmatism (Alcon), Biofinity ${ }^{\circledR}$ Toric (CooperVision), Acuvue ${ }^{\circledR}$ advance for astigmatism (Vistakon), and Proclear $^{\circledR}$ Toric (CooperVision). The first four contact lenses were silicone-hydrogel and the last one was a traditional hydrogel. The characteristics and parameters of each lens [6] are shown in Table 1.

Table 1: Parameters of study lenses

\begin{tabular}{|c|c|c|c|c|c|}
\hline Product name & $\begin{array}{l}\text { Pure Vision } \\
\text { Toric }\end{array}$ & $\begin{array}{c}\text { Acuvue } \\
\text { advance for } \\
\text { astigmatism }\end{array}$ & $\begin{array}{l}\text { Air optix for } \\
\text { astigmatism }\end{array}$ & Biofinity Toric & Proclear Toric \\
\hline Manufacturer & $\begin{array}{c}\text { Bausch \& } \\
\text { Lomb }\end{array}$ & Vistakon & Alcon & Cooper Vision & Cooper Vision \\
\hline DK & 91 & 60 & 110 & 128 & 27 \\
\hline $\mathrm{DK} / \mathrm{t}$ & 91 & 86 & 108 & 116 & 25 \\
\hline CT@-3.00(mm) & 0.1 & 0.07 & 0.102 & 0.11 & 0.11 \\
\hline $\begin{array}{c}\text { Water content } \\
(\%)\end{array}$ & 36 & 47 & 33 & 48 & 62 \\
\hline Base curve $(\mathrm{mm})$ & 8.70 & 8.60 & 8.70 & 8.70 & 8.5 \\
\hline Diameter (mm) & 14.0 & 14.5 & 14.50 & 14.50 & 14.0 \\
\hline $\begin{array}{l}\text { Lens axis } \\
\text { marking (s) }\end{array}$ & $\begin{array}{l}\text { At } 5,6,7 \\
\text { o'clock }\end{array}$ & $\begin{array}{l}\text { At } 6,12 \\
\text { o'clock }\end{array}$ & $\begin{array}{l}\text { At 3, 6, } 9 \\
\text { o'clock }\end{array}$ & One at 6 o'clock & $\begin{array}{l}3 \text { radial lines } \\
\text { at } 6 \text { o'clock } \\
\text { and } 15 \\
\text { degree either } \\
\text { side }\end{array}$ \\
\hline $\begin{array}{l}\text { Surface } \\
\text { treatment }\end{array}$ & $\begin{array}{l}\text { Plasma } \\
\text { oxidation }\end{array}$ & $\begin{array}{c}\text { Internal } \\
\text { wetting } \\
\text { agent }\end{array}$ & $\begin{array}{l}\text { Plasma } \\
\text { treatment }\end{array}$ & $\begin{array}{l}\text { Inherently } \\
\text { wettable }\end{array}$ & $\begin{array}{c}\text { PC } \\
\text { Technology }\end{array}$ \\
\hline Material & Balafilcon A & Galyfilcon A & Lotrafilcon B & Comfilcon A & Omafilcon \\
\hline Modulus & 1.5 & 0.43 & 1.00 & 0.8 & Low \\
\hline Design & $\begin{array}{l}\text { Back surface } \\
\text { toric, Prism } \\
\text { ballast (Lo- }\end{array}$ & $\begin{array}{c}\text { Back surface } \\
\text { toric, } \\
\text { accelerated }\end{array}$ & $\begin{array}{l}\text { Back surface } \\
\text { toric, modified } \\
\text { prism ballast }\end{array}$ & $\begin{array}{l}\text { Toric design, } \\
\text { modified prism } \\
\text { ballast design }\end{array}$ & $\begin{array}{c}\text { Back surface } \\
\text { toric, prism }\end{array}$ \\
\hline
\end{tabular}




\begin{tabular}{|l|l|l|l|l|l|}
\hline & torque) & $\begin{array}{c}\text { stabilization } \\
\text { design } \\
\text { (ASD) }\end{array}$ & $\begin{array}{c}\text { design (8/4 } \\
\text { precision } \\
\text { balance } \\
\text { design) }\end{array}$ & $\begin{array}{c}\text { (Equi ballast or } \\
\text { Horizontal iso- } \\
\text { thickness } \\
\text { design) }\end{array}$ & ballast \\
\hline
\end{tabular}

${ }^{\star}$ DK unit: $\left(\mathrm{cm}^{2} / \mathrm{sec}\right)\left(\mathrm{mlO}_{2} / \mathrm{ml} \times \mathrm{mmHg}\right) 10^{-11}$

Subjects were fitted with the various toric contact lenses in a randomized order. The selected lens was fitted and the initial assessment was performed after five minutes. The assessed fitting characteristics using the slit lamp biomicroscope were full corneal coverage in all positions of gaze with about $1 \mathrm{~mm}$ conjunctival overlap and good centration. Following these tasks, the dynamic assessment (post blink and version lag movements and the push-up test) was performed. After a settling time of 20 minutes, the subject positioned behind the slit lamp and gazed into primary position, the location of the toric lens marking(s) was identified for recording of lens orientation and rotational recovery.[11]

Lens orientation was evaluated by narrowing the width of the slit lamp beam to approximately $0.5 \mathrm{~mm}$ and focusing on the lens surface at the 6 o'clock position on the lens axis marking(s). The vertical beam was slowly rotated to align lens marking and measure the amount of lens rotation. Rotation was considered as the number of degrees the lens moves from the vertical position in either direction. It was measured three times and the average was recorded in degrees. To improve accuracy in measurement the light beam was passed through the center of the pupil.[6,11]

Rotational recovery was assessed after determining the position of the lens axis marking(s) for each of the study contact lenses in primary position. While slit lamp magnification was set at 10x magnification, the contact lens was manually rotated $45^{\circ}$ inferotemporally from primary gaze orientation. The subjects were asked to blink naturally and then allowing the lens to recover to its initial position. Time required to return to the primary gaze position was recorded in second as rotational recovery.[5, 6]

The above assessments were repeated separately for each lens. After completing assessments with each lens, the lens was removed. The subject was provided a 10 min rest period, before the next lens was trialed. 
The right eye only was chosen for this evaluation. All lenses had the same power $1.00 /-1.75 \times 90 .[2]$

Data were analyzed in SPSS.17 software (SPSS for Windows, SPSS Inc. Chicago, IL, USA). Normality of data was assessed with the Kolmogorov-Smirnov test which indicated normal distribution. The Repeated Measures ANOVA and Pearson correlation tests were used for statistical analysis. In all tests, the significance level was considered to be statistically significant is $p<0.05$.

\section{Results}

From the 20 students in this study, 15 were female and 5 male. The mean ages in all subjects and separately in females and males were $21.4 \pm 2.0$ years, $21.3 \pm 2.2$ years, $21.6 \pm 1.1$ years, respectively.

Biometric data of the subjects is shown in Table 2.

Table 2: Subject's biometric data

\begin{tabular}{|c|c|c|}
\hline \multicolumn{1}{|c|}{ Statistics } & Mean \pm SD & $95 \% \mathrm{C} 1$ \\
\hline Spheriables & & \\
\hline Cylindrical refractive error (D) & $-1.37 \pm 1.90$ & -2.26 to -0.48 \\
\hline K flat (D) & $42.94 \pm 1.68$ & -1.80 to -1.06 \\
\hline K steep(D) & $44.12 \pm 1.97$ & 43.19 to 43.73 \\
\hline K flat - K steep(D) & $-1.17 \pm 0.93$ & -1.60 to -0.73 \\
\hline
\end{tabular}


The mean ( $\pm 1 \mathrm{SD}$ ) values for the lens rotation from the vertical position and rotational recovery to primary gaze orientation following a $45^{\circ}$ manual misorientation are presented in Table 3.

Table 3: Mean and SD of lens rotation from the vertical position and rotational recovery for different contact lenses

\begin{tabular}{|c|c|c|c|c|c|}
\hline \multirow{2}{*}{ Vontact Lens } & PVT & AO & AAfA & BT & PT \\
\cline { 2 - 6 } & Mean \pm SD & Mean \pm SD & Mean \pm SD & Mean \pm SD & Mean \pm SD \\
$(95 \% \mathrm{C} 1)$ & $(95 \% \mathrm{C} 1)$ & $(95 \% \mathrm{C} 1)$ & $(95 \% \mathrm{C} 1)$ & $(95 \% \mathrm{C} 1)$ \\
\hline $\begin{array}{c}\text { Misorientation } \\
\text { Rotation }\end{array}$ & $6.00 \pm 5.52$ & $5.50 \pm 5.35$ & $6.85 \pm 4.93$ & $2.25 \pm 4.12$ & $8.75 \pm 8.56$ \\
(Degree) & $(3.41-8.58)$ & $(2.99-8.00)$ & $(4.54-9.15)$ & $(0.31-4.18)$ & $(4.72-12.75)$ \\
\hline $\begin{array}{c}\text { Rotational } \\
\text { Recovery }\end{array}$ & $73.90 \pm 49.49$ & $60.20 \pm 35.68$ & $94.20 \pm 64.95$ & $28.50 \pm 16.53$ & $89.25 \pm 59.84$ \\
(Second) & $(50.73-97.06)$ & $(43.49-$ & $(63.79-$ & $(20.76-$ & $(61.24-$ \\
& & $76.90)$ & $124.60)$ & $36.24)$ & $117.25)$ \\
\hline
\end{tabular}

PVT: Pure Vision Toric; AO: Air Optix for Astigmatism; AAfA: Acuvue Advance for Astigmatism; BT: Biofinity Toric; PT: Proclear Toric

Table 3 shows that the lowest lens rotation was from the Biofinity Toric and the highest misorientation was from the Proclear Toric.

The repeated measurement ANOVA was used for comparison of lens rotation among different contact lenses and showed significant differences among them. 
$(F(2.12,40.29)=6.19, P=0.004)$ The Bonferroni adjustment was used for pairwise comparisons. The test results indicated that this difference was only between the Biofinity Toric with other lenses (PVT: $P=0.02, A O: P=0.02$, AAfA: $P=0.005, P T$ : $P=0.04)$ while in other pairs there was not a statistically significant difference $(\mathrm{P}>0.05)$.

Figure 1 takes into account the direction of lens rotation from the vertical position or zero position in either direction. Three conditions were considered: zero position, Inferonasal and inferotemporal orientations.

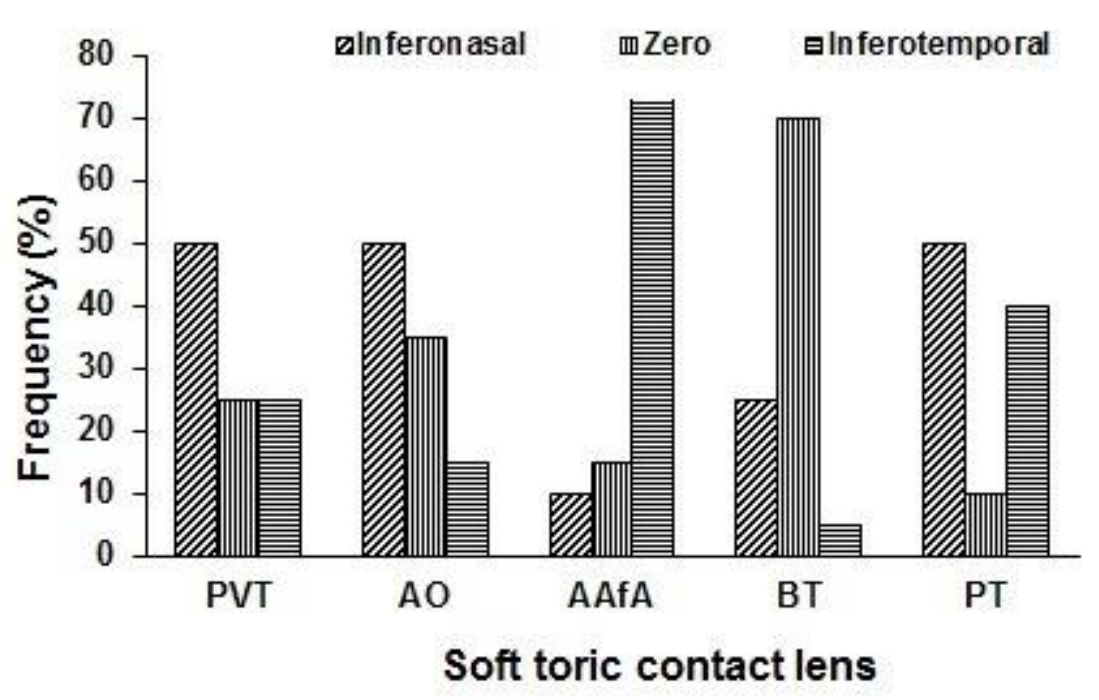

Figure 1: Frequency of different orientations from the vertical position for different lenses (PVT: Pure Vision Toric; AO: Air Optix for Astigmatism; AAfA: Acuvue Advance for Astigmatism; BT: Biofinity Toric; PT: Proclear Toric)

Also, the above table illustrates the highest and the lowest reorientation speed following a $45^{\circ}$ manual temporalward misorientation from primary gaze orientation were related to the Biofinity Toric and the Acuvue Advance for Astigmatism, respectively.

There was a significant difference using the repeated measurement ANOVA in comparison of reorientation speed among different contact lenses. $(F(4,76)=6.60$, $\mathrm{P}<0.001)$ The Bonferroni test indicated that this difference was only between the 
Biofinity Toric with other lenses (PVT: $P=0.005, A O: P=0.02$, AAfA: $P=0.003$, PT: $P=0.004)$ while in other pairs there was not a statistically significant difference. $(P>0.05)$

\section{Discussion}

The results of this study showed that stabilization technique can influence on the lens orientation in the eye so that the lowest and the highest lens rotation from the vertical position in the current study were related to the Biofinity Toric (CooperVision) and the Proclear Toric (CooperVision), respectively. Statistically significant difference in lens rotation was only found for the Biofinity Toric (CooperVision) lens with other lenses [PureVision Toric (B\&L), Air Optix for Astigmatism (Alcon), Proclear Toric (CooperVision), and Acuvue advance for astigmatism (Vistakon)].

In designing of the Biofinity Toric lenses a modified prism ballast design called Optimized Ballast or Equi Ballast or Horizontal iso-Thickness has been used.

The literature suggests that there will be an inappropriate fitting if the lens rotation is more than 30 degrees from the intended position.[11] Hence, each of the study lenses provides appropriate position orientation although the optimized ballast method compared with other techniques [classic prism ballast (Pure Vision Toric, Proclear Toric), modified prism ballast (Air optix for astigmatism), and accelerated stabilization design (Acuvue advance for astigmatism)] provides the best lens orientation on the eye lens and finally the individual's visual performance. Due to lesser lens rotation from the desired orientation and reduce of the need to re-order, the examiner can fit this lens with more confidence.

While the stabilization method employed by the Pure Vision Toric, Proclear Toric, Air optix for astigmatism, and Acuvue advance for astigmatism did not show significant difference in the lens orientation on the eye that is in contrast with the findings of Cairns et al. (2009) although they evaluated only two of lenses used in the present study (Pure Vision Toric and Acuvue advance for astigmatism).[12]

Their reported mean of rotation for the Pure Vision Toric and Acuvue advance for astigmatism contact lenses were $11.6 \pm 9.4$ and $7.2 \pm 8.9$ degrees, respectively that 
were not only different with values of the present study $(6.00 \pm 5.52$ degree for the Pure Vision Toric and 6.85 \pm 4.93 degrees for the Acuvue advance for astigmatism) but also they obtained more rotation with the Pure Vision Toric lenses compared to the Acuvue advance for astigmatism, whereas the opposite was found in the present study. These differences can partly be attributed to the fit assessment's time. (3 minutes versus 20 minutes in the present study).

Ideally, the fit should be assessed briefly after a few minutes and then after at least 15 minutes.[6] This settling time is critical firstly because lens equilibration with tear film and secondly, change of the upper eyelid action on the lens's thickness profile over the first several minutes as the lens rotates to its preferred orientation. Therefore, the most effective time to predict the final fitting characteristics is 15 to 20 minutes after lens insertion.

Young et al. (2009) reported the highest lens rotation for the PureVision Toric (B\&L) and in decreasing trend for Proclear Toric, Acuvue advance for astigmatism, Air Optix for Astigmatism [4] which is in contrast with the obtained sequence in the current investigation. (Proclear Toric > Acuvue Advance for Astigmatism > Pure Vision Toric > Air Optix for Astigmatism) Also, the present study did not show difference in the stabilization techniques used in these four lenses in contrast to their study. Although there is a common point in the two studies that the modified ballast methods used in the Air Optix for Astigmatism lens allocated the best orientation to it among these four lenses.

Better orientation at the primary position of gaze using the ballast techniques compare to the non-prism ballast (accelerated stabilization) method was also mentioned by Tan et al. (2007) [3] which is in agree to the results of this study.

With respect to rotational recovery, the optimized ballast technique used in the design of Biofinity Toric lenses showed the highest speed and the accelerated stabilization design used in Acuvue advance for astigmatism lenses indicated the lowest reorientation speed following a $45^{\circ}$ manual inferotemporal misorientation from primary gaze orientation.

Also, the modified ballast design showed higher speed than the classic ballast method. However, the only difference was between the optimized ballast designs 
with other designs statistically. The difference between the Biofinity Toric lens and the Proclear Toric besides difference in the stabilization techniques can partly be attributed to the fact that although these two lenses have the same power, they do not have the same base curve, with the Proclear lens providing the steepest fit and the actual fit (flat versus steep) may influence on rotational stability. The current findings are in agreement with the results of Cairns et al. (2009), they also have more recovery time reported with the Acuvue advance for astigmatism lens compared to the Pure Vision Toric lens.[12] The obtained values are not same in two studies because of different assessment methods. They manually rotated the lens $45^{\circ}$ temporally from primary gaze orientation and allowing one minute for the lens to recover and the absolute difference between the primary and final positions of lens was taken as the rotational recovery. While as in the present study, time required to return to the primary gaze position following the manually rotation $45^{\circ}$ inferotemporally from primary orientation was recorded as rotational recovery. Although, there was a significant difference in the rotational recovery in their study but this was not seem in this study.

The results of Young et al. (2009) also confirm the results of the present study that the Pure Vision Toric, Proclear Toric, Air Optix for Astigmatism, and Acuvue advance for astigmatism lenses did not differ in their rotational recovery [4] although the obtained recovery speed sequence in the present study contrasts with theirs.

Disclosure: The authors report no conflicts of interest and have no proprietary interest in any of the materials mentioned in this article.

Acknowledgements: This research was supported by the health promotion research center of Zahedan University of Medical Sciences. The authors would like to thank Dr. Alireza Ansari-Moghaddam for assistance in statistical analysis and the participants who made this study possible. 


\section{References}

[1] Young G, Veys J, Pritchard N, Coleman S. A multi-centre study of lapsed contact lens wearers. Ophthalmic Physiol Opt 2002; 22:516-27.

[2] Tan J, Papas E, Carnt N, et al. Performance Standards for Toric Soft Contact Lenses. Optom Vis Sci 2007; 84:422-8.

[3] Young G, Mcllraith R, Hunt C. Clinical Evaluation of Factors Affecting Soft Toric Lens Orientation. Optom Vis Sci 2009; 86:1259-66.

[4] Young G, Hunt C, Covey M. Clinical Evaluation of Factors Influencing Toric Soft Contact Lens Fit. Optom Vis Sci 2002; 79:11-9.

[5] Fonn D, Dumbleton K, Jones L, du Toit R, Sweeney D. Silicone Hydrogel Material and Surface Properties. Contact Lens Spectrum 2002; 17:24-8.

[6] Bennett E, Henry VA. Clinical Manual of Contact Lenses. $3^{\text {nd }}$ edition, Philadelphia: Lippincott Williams and Wilkins; 2012. p. 345-57.

[7] Goldsmith WA, Steel S. Rotational Characteristics of Toric Contact Lenses. ICLC 1991; 18:227-9.

[8] Andrzejewski TM, Pence N. Design, Materials, and Fitting of Toric Silicone Hydrogel Lenses. Contact Lens Spectrum 2011.

[9] Gasson A, Morris JA. The Contact Lens Manual: A Practical Guide to Fitting. 3rd edition, Oxford; Boston: Butterworth-Heinemann; 2003. p. 68, 70.

[10] Jones L, Subbaraman LN, Dumbelton K, Rogers R. Surface Treatment, Wetting and Modulus of Silicon Hydrogels. Optician 2006; 232:28-44.

[11] Zikos G, Kong S, Ciuffreda K, Selenow A, et al. Rotational Stability of Toric Soft Contact Lenses During Natural Viewing Conditions. Optom Vis Sci 2007; 84:103945.

[12] Cairns G, China P, Green T, Reindel B. Differences in toric lens performance: lens orientation and orientation recovery. American Academy of Optometry. 2009. Poster presentation. 